\title{
Utilização de Modelo Network DEA na Avaliação de Cursos de Pós-Graduação Stricto Sensu em Engenharia
}

\author{
Silvio Figueiredo Gomes Júnior \\ Fundação Centro Universitário Estadual da Zona Oeste \\ Av. Manuel Caldeira de Alvarenga, 1203, Campo Grande, 23070-200, Rio de Janeiro, RJ \\ silviogomes@uezo.rj.gov.br \\ Plácido Moreno Beltrán \\ Departamento de Organización Industrial y Gestión de Empresas I - Universidad de Sevilla \\ Escuela Técnica Superior de Ingeniería. Camino de los Descubrimientos s/n. 41092. Spain. \\ placidomb@us.es \\ João Carlos Correia Baptista Soares de Mello \\ Departamento de Engenharia de Produção - Universidade Federal Fluminense \\ Rua Passo da Pátria 156, São Domingos, 24210-240, Niterói, RJ \\ jcsmello@producao.uff.br \\ Lidia Angulo Meza \\ Dep. de Engenharia de Produção - Universidade Federal Fluminense \\ Av. dos Trabalhadores, 420, 27255-125, Volta Redonda, RJ \\ lidia@metal.eeimvr.uff.br
}

Resumo

Os cursos de pós-graduação stricto sensu no Brasil são avaliados a cada três anos. Os métodos utilizados nesta avaliação buscam medir a produtividade acadêmica (incluindo a quantidade e qualidade da produção científica), o número de estudantes que concluem o curso, entre outros fatores. Assim, o objetivo deste trabalho é avaliar a capacidade dos programas de pós-graduação da área de Engenharia III da CAPES na produção e publicação de artigos científicos de mestrados e teses de doutorado. Foi utilizado um modelo de Análise Envoltória de Dados (DEA) em dois estágios, denominado NDEA, onde o número de dissertações e teses são os produtos intermediários. Desta forma, é possível calcular a eficiência tanto nos aspectos acadêmicos quanto nos aspectos de pesquisa dos cursos de pós-graduação.

Palavras-chave: CAPES, Network DEA, Avaliação Educacional.

Abstract

Brazilian post-graduate courses are evaluated every three years. The methods used in this evaluation try to measure academic productivity (including quantity and quality of scientific production), number of students concluding the course, among other factors. Therefore, the aim of this work is to evaluate the ability of post-graduate Engineering III programmes of CAPES areas to produce scientific papers from masters' degrees and doctoral theses in a proper way. We have implemented a two-stage Network Data Envelopment Analysis (NDEA) model using the number of thesis as an intermediate variable. The model allows us to compute the efficiencies from both the academic and research aspects of the post-graduate courses.

Keywords: CAPES, Network DEA, Educational evaluation. 


\section{INTRODUÇÃO}

Desde 1976, os cursos de pós-graduação stricto sensu no Brasil vem sendo avaliados pela CAPES (Coordenação de Aperfeiçoamento de Pessoal de Nível Superior). Esta avaliação compreende, entre outros aspectos, a realização do acompanhamento anual e da avaliação trienal do desempenho de todos os programas e cursos que integram o Sistema Nacional de Pósgraduação (SNPG). Os resultados desse processo são expressos em notas na escala de "1" a "7" e fundamentam a obtenção da renovação de "reconhecimento" do curso, a vigorar no triênio subsequente.

É importante ressaltar que as avaliações de produtividade na educação são normalmente subjetivas, devido ao grande número de variáveis a serem levadas em consideração. Assim, é necessário atribuir pesos para quantificar essas variáveis e agregá-las em um único índice. Esta subjetividade no processo de avaliação pode causar mal-estar e produzir resultados que podem ser rejeitados pelos leitores.

De acordo com Boclin (1999), a avaliação educacional deveria ser quantitativa e comparável. Neste sentido, a metodologia Análise Envoltória de Dados (DEA) (Cooper et al., 2000) se mostra bastante adequada pois possui essas duas características simultaneamente. Os pesos atribuídos pelos modelos DEA tradicionais são calculados por meio de um problema de programação linear, de tal forma que cada decisão beneficia a melhor combinação possível destes pesos, com propósito de maximizar a sua eficiência (Thanassoulis et al., (2004). Por outro, estes pesos podem não ser considerados "justos" por todos os programas. No entanto, ao fornecer uma certa "liberdade" para determinar estes pesos, dá-se a oportunidade para que cada programa destaquem o que tem de melhor. Neste sentido, os programas que são ineficientes nestas condições realmente não tem bom desempenho em qualquer dos quesitos avaliados pelo CAPES.

Propõe-se neste trabalho a utilização de um modelo DEA em dois estágios chamado Network DEA com o objetivo de avaliar a capacidade dos docentes dos programas de pós-graduação da área de Engenharia III da CAPES em orientar alunos nos programas de mestrado e doutorado e fazer com que os mesmos publiquem seus trabalhos. Nesse modelo, as opinões subjetivas, consensuais na maioria dos docentes, são expressas por meio de restrições aos pesos.

Ao contrário dos modelos DEA tradicionais, o modelo Network DEA (NDEA) define cada DMU como uma rede de sub-processos. Ou seja, considera a estrutura interna do sistema e permite o cálculo das ineficiências correspondentes aos diferentes estágios, assim como considera também suas respectivas eficiências, ou seja, decompõe a eficiência global em estágios específicos. De acordo com o modelo NDEA, cada sub-processo pode ter diferentes inputs (variáveis de entrada) e outputs (variáveis de saída) exogenos. No entanto, os estágios internos de uma DMU estão conectados através de variáveis intermediárias que são endógenas. Assim, no modelo NDEA deste trabalho, é usado o número de dissertações e teses são os produtos intermediários, uma vez que o produto final deve ser a publicação de artigos científicos. Desta forma, o modelo permite calcular a eficiência tanto nos aspectos de produtividade quanto qualidade de pesquisa dos cursos de pós-graduação. Neste artigo, os dois modelos de produtividade e qualidade de Soares de Mello et al. (2006) são agregados em um único modelo DEA. Foram utilizados os dados do último triênio de avaliação (2010-2012), divulgados pela Capes.

O artigo está organizado da seguinte forma: a seção 2 descreve uma revisão da literatura tanto nos aspectos de DEA em educação quanto em relação aos modelos Network DEA. A seção 3 
descreve o modelo Network DEA e apresenta sua formulação. A seção 4 descreve a modelagem utilizada no trabalho. A seção 5 apresenta os resultados e discussões e a seção 6 traz as conclusões do trabalho.

\section{REVISÃO DA LITERATURA}

\subsection{Revisão de DEA em Educação}

Charnes et al. (1978) publicaram o primeiro artigo sobre DEA, que foi em avaliação educacional. Inicialmente, DEA foi desenvolvido para avaliar organizações sem considerar aspectos financeiros, focando, por exemplo, os aspectos educacionais.

Desde esta publicação, diversos artigos e técnicas em DEA vem sendo desenvolvidas em avaliações educacionais. Soares de Mello et al. (2006) avaliaram os programas de pós-graduação da COPPE (Instituto de Pós-graduação e Pesquisa de Engenharia) da Universidade Federal do Rio de Janeiro e fizeram uma revisão da literatura de DEA em educação até 2005. Angulo Meza et al. (2011) utilizaram DEA e redes neurais de Kohonen para avaliar as melhores localizações para instalação de polos do CEDERJ (Centro de Educação Superior à Distância do Estado do Rio de Janeiro) e Angulo Meza et al. (2012) utilizaram DEA e modelo multiobjetivo na avaliação da eficiência dos polos de educação superior à distância do CEDERJ.

Thieme et al. (2012) analisaram a performance de 54 países que participaram do programa PISA 2006 (Programme for International Student Assessment) e Gomes Júnior et al. (2013) mostraram a utilização de índices não radiais em DEA e mostram sua aplicação na identificação de alvos não radiais para os polos do CEDERJ e o cálculo da eficiência não radial em relação a estes polos. Vários outros trabalhos utilizam esta metodologia para cálculo de eficiência ou benchmarks para professores, cursos e instituições de ensino. Por exemplo, pode-se citar Tyagi et al. (2009), França et al. (2010), Bougnol e Dulá (2006), entre outros.

\subsection{Revisão Network DEA}

O modelo NDEA inicial foi desenvolvido por Färe e Grosskopf (2000), que propuseram a abertura da chamada "caixa preta". No entanto, os primeiros trabalhos utilizando NDEA buscavam a solução de um modelo DEA para cada etapa de forma independente, desconsiderando a continuidade das relações entre os nós. Entre estes trabalhos, pode-se citar Seiford e Zhu (1999), Zhu (2000), Sexton e Lewis (2003), Chilingerian e Sherman (2004), Narasimhan et al. (2004) e Soares de Mello et al. (2006).

Chen e Zhu (2004) propuseram um modelo DEA com retornos constantes de escala que deixasse de considerar as eficiências de cada estágio de forma independente. Mais recentemente, Kao (2009) levou em conta a relação entre as etapas com a abordagem NDEA relacional, enquanto Chen et al. (2009) propuseram um modelo de NDEA considerando a continuidade dos produtos intermédios. Em seguida, Tone e Tsutsui (2009) adaptaram o modelo SBM (Slack-Based Measure) ao modelo NDEA, permitindo a identificação de ineficiências não radiais. Uma aplicação deste modelo pode ser encontrada em Moreno e Lozano (2014).

É importante destacar que, até o momento, não foram encontradas aplicações dos modelos relacionais de Network DEA em educação como é feita neste trabalho.

\section{MODELO NETWORK RELACIONAL MULTIPLICATIVO DEA}

Os modelos DEA tradicionais (por exemplo, CCR desenvolvido por Charnes et al. (1978) e BCC desenvolvido por Banker et al. (1984)) tem-se mostrado adequados para a análise de eficiência das unidades de produção que utilizam múltiplos inputs (insumos) e múltiplos outputs (produtos). Estas unidades de produção são, geralmente, chamadas de unidades tomadoras de decisão, ou 
simplesmente DMU (decision making units). A eficiência é calculada para cada DMU, comparando os níveis de inputs e outputs de cada DMU com todas as outras.

Como destacado anteriormente, uma das limitações dos modelos DEA tradicionais é negligenciar os estágios internos de uma DMU e as atividades de ligação (produtos intermédios) entre eles. Na verdade, a existência de conexão entre as atividades é uma característica indispensável dos modelos Network DEA (NDEA). Embora os modelos NDEA possam ser encontrados em diversas configurações, o modelo serial de multi-estágio é o mais comum. O diagrama de produção de um processo com três estágios hipotéticos é ilustradro na Figura 1.

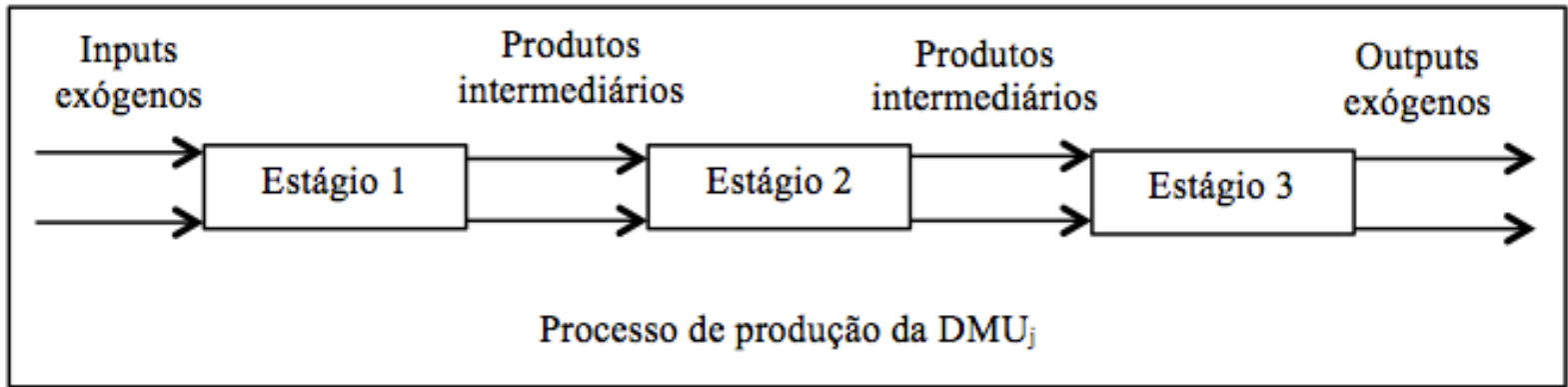

Figura 1 - Representação de um sistema serial com três estágios.

Devido à sua estrutura, para que uma DMU possa ser eficiente nos modelos NDEA, esta deve ser eficiente em cada sub-processo (ou estágio). Assim, pode ocorrer de não existir uma DMU que seja totalmente eficiente (isto é, eficiência igual a 100\%) quando se considera todo o processo. Nesta modelagem, os produtos intermediários são produzidos e consumidos na DMU, ao passo que os inputs e outputs são exógenos à estrutura interna. Além do cálculo global da eficiência, os modelos NDEA fornecem os índices de eficiência de cada estágio, uma vez que o conjunto de possibilidades de produção é modelado no nível de cada estágio, ou seja, cada sub-processo tem sua própria tecnologia, definindo assim o seu próprio conjunto de pesos e unidades de referência.

Kao and Hwang (2008) apresentaram o modelo relacional multiplicativo. Este modelo é baseado no modelo DEA com retorno constante de escala, onde $E_{0}$ é a eficiência do problema de programação fracionária descrito em (1).

$$
\begin{aligned}
& E_{0}=\max \frac{\prod_{r=1}^{s} u_{r} \cdot y_{r 0}}{\prod_{i=1}^{m} v_{i} \cdot x_{i 0}} \\
& \text { s.t. } \frac{\prod_{r=1}^{s} u_{r} \cdot y_{r j}}{\prod_{i=1}^{m} v_{i} \cdot x_{i j}} \square 1, j=1,2, \ldots, n . \\
& u_{r}, v_{i} \square \square, i=1,2, \ldots, m ; r=1,2, \ldots, s .
\end{aligned}
$$

As eficiências $E_{0}^{1}$ e $E_{0}^{2}$ referentes aos estágios 1 e 2, respectivamente, são calculadas pelos modelos (2) e (3), baseados no modelo DEA CRS apresentado em (1): 


$$
\begin{aligned}
& E_{0}^{1}=\max \frac{\prod_{d=1}^{D} w_{d} \cdot z_{d 0}}{\prod_{i=1}^{m} v_{i} \cdot x_{i 0}} \\
& \text { s.t. } \frac{\prod_{d=1}^{D} w_{d} \cdot z_{d j}}{\prod_{i=1}^{m} v_{i} \cdot x_{i j}} 1, j=1,2, \ldots, n . \\
& w_{d}, v_{i} \square \square, i=1,2, \ldots, m ; d=1,2, \ldots, D . \\
& E_{0}^{2}=\max _{r=1}^{s} u_{r} \cdot y_{r 0} \\
& \prod_{d=1}^{D} w_{d} . z_{d 0} \\
& \text { s.t. } \frac{\prod_{r=1}^{D} u_{r} \cdot y_{r j}}{\prod_{d=1}^{D} w_{d} . z_{d j}} \square 1, j=1,2, \ldots, n . \\
& u_{d} \square \square, d=1,2, \ldots, D ; r=1,2, \ldots, s .
\end{aligned}
$$

De acordo com Kao and Hwang (2008), a eficiência global $E_{0}$ e as eficiências individuais $E_{0}^{1} \mathrm{e}$ $E_{0}^{2}$ referentes aos estágios 1 e 2 da DMU em análise é dada pelas equações (4), (5) e (6) respectivamente.

$$
\begin{aligned}
& E_{0}=\frac{\prod_{r=1}^{s} u_{r}^{*} \cdot y_{r 0}}{\prod_{i=1}^{m} v_{i}^{*} \cdot x_{i 0}} \square 1 \\
& E_{0}^{1}=\frac{\prod_{d=1}^{D} w_{d}^{*} \cdot z_{d 0}}{\prod_{i=1}^{m} v_{i}^{*} \cdot x_{i 0}} \square 1 \\
& E_{0}^{2}=\frac{\prod_{r=1}^{s} u_{r}^{*} \cdot y_{r 0}}{\prod_{d=1}^{D} w_{d}^{*} \cdot z_{d 0}}
\end{aligned}
$$

Onde $u_{r}^{*}, v_{i}^{*}$ e $w_{p}^{*}$ são os pesos ótimos calculados pelos modelos (2) e (3). Assim, a eficiência global (7) é o produto das eficiências individuais de cada estágio. 


$$
E_{0}=E_{0}^{1} x E_{0}^{2}
$$

De forma a incorporar a interação entre os 2 estágios, Kao and Hwang (2008) incluíram as restrições (4), (5) e (6) no modelo (1), descrito em (8).

$$
\begin{aligned}
& E_{0}=\max \frac{\prod_{r=1}^{s} u_{r} \cdot y_{r 0}}{\prod_{i=1}^{m} v_{i} \cdot x_{i 0}} \\
& \text { s.t. } \frac{\prod_{r=1}^{s} u_{r} \cdot y_{r j}}{\prod_{i=1}^{m} v_{i} \cdot x_{i j}} \square 1, j=1,2, \ldots, n . \\
& \frac{\prod_{d=1}^{D} w_{d} \cdot z_{d j}}{\prod_{i=1}^{m} v_{i} \cdot x_{i j}} \square 1, j=1,2, \ldots, n . \\
& \frac{\prod_{r=1}^{s} u_{r} \cdot y_{r j}}{\prod_{d=1}^{D} w_{d} \cdot z_{d j}} \\
& u_{r}, v_{i}, w_{d} \square \square, i=1,2, \ldots, m ; r=1,2, \ldots, s ; d=1,2, \ldots, D .
\end{aligned}
$$

Além disso, os autores consideraram os mesmos pesos para os produtos intermediários, independentemente se estes produtos intermediários são outputs do estágio 1 ou inputs do estágio 2. Esta suposição faz a ligação entre os 2 estágios e permite que o modelo fracionário (8) seja convertido no modelo de programação linear (9).

$$
\begin{aligned}
& E_{0}=\max \prod_{r=1}^{s} u_{r} \cdot y_{r 0} \\
& \text { s.t. } \prod_{i=1}^{m} v_{i} \cdot x_{i 0}=1 \\
& \prod_{r=1}^{s} u_{r} \cdot y_{r j} \square \prod_{i=1}^{m} v_{i} \cdot x_{i j} \square 0, j=1,2, \ldots, n . \\
& \prod_{d=1}^{q} w_{d} \cdot z_{d j} \square \prod_{i=1}^{m} v_{i} \cdot x_{i j} \square 0, j=1,2, \ldots, n . \\
& \bigsqcup_{r=1}^{s} u_{r} \cdot y_{r j} \square \prod_{d=1}^{q} w_{d} \cdot z_{d j} \square 0, j=1,2, \ldots, n . \\
& u_{r}, v_{i}, w_{d} \square \square, i=1,2, \ldots, m ; r=1,2, \ldots, s ; d=1,2, \ldots, D .
\end{aligned}
$$


Os pesos ótimos do modelo (9) podem não ser únicos, assim como a decomposição da eficiência global $E_{0}$ nas eficiências de cada estágio $E_{0}^{1}$ e $E_{0}^{2}$ podem também não serem únicas. Kao and Hwang (2008) propuseram a maximização de uma das eficiências individuais, por exemplo $E_{0}^{1}$, enquanto mantém a eficiência global $E_{0}$ calculada pelo modelo (9). Assim, a outra eficiência individual $E_{0}^{2}$ é calculada pela expressão (10).

$$
E_{0}=E_{0}^{1} x E_{0}^{2} \square E_{0}^{2}=\frac{E_{0}}{E_{0}^{1}}
$$

\section{MODELAGEM}

Na avaliação da qualidade dos cursos de pós-graduação, pretende-se avaliar a capacidade de cada programa em publicar o desenvolvimento de suas pesquisas, tanto ao nível de mestrado quanto ao nível de doutorado. Se um programa possui um baixo índice de publicação de suas pesquisas, o mesmo é considerado de baixa qualidade. Por outro lado, se um programa possui um elevado número de trabalhos publicados (ou outra produção acadêmica), principalmente em jornais internacionais bem reconhecidos, é uma indicação da alta qualidade do programa.

Assim, o objetivo deste trabalho é avaliar a capacidade dos docentes dos programas de pósgraduação da área de Engenharia III da CAPES em orientar alunos nos programas de mestrado e doutorado e fazer com que os mesmos publiquem seus trabalhos. Para esta avaliação, foi utilizado um modelo NDEA multiplicativo relacional de dois estágios descrito na seção 3 onde as DMUs do modelo são os programas de pós-graduação que tiveram defesas de mestrado ou de doutorado defendidas no triênio 2010-2012. A variável de input inicial é o número de docentes dos programas de pós-graduação. O número de dissertações de mestrado e teses de doutorado são os produtos intermediários e os outputs finais do processo são as publicações de artigos científicos em periódicos científicos avaliados pela CAPES, num total de 7 (sete) outputs referentes às qualificações QUALIS dos periódicos (A1, A2, B1, B2, B3, B4 e B5). Desta forma, o modelo nos permite calcular a eficiência tanto nos aspectos acadêmicos quanto nos aspectos de pesquisa dos programas de pós-graduação, pois fornece a eficiência destes programas em cada uma das etapas (na produção das dissertações de mestrado e teses de doutorado no primeiro estágio e na produção dos artigos científicos no segundo estágio), assim como a eficiência global destes programas. A Figura 2 apresenta o esquema do processo de produção de uma DMU.

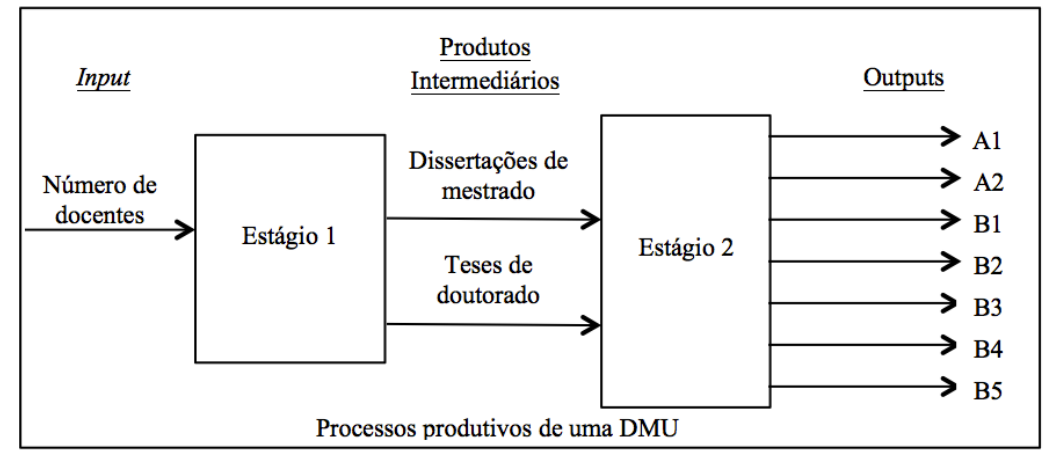

Figura 2 - Representação do sistema de 2 estágios

Vale destacar que a limitação temporal do modelo foi feita para manter coerência com a avaliação da CAPES, que leva em conta as publicações e defesas do mesmo triênio. A hipótese assumida neste trabalho é que há uma certa estabilidade na geração temporal de publicações pelas teses e dissertações, implicitamente também assumida pela CAPES na sua avaliação pois, na 
verdade, não há como saber quando uma tese irá gerar um artigo, podendo ser antes ou depois da sua defesa.

O primeiro estágio está relacionado com a capacidade dos docentes dos programas (input exógeno) em orientar seus alunos na produção e defesa das dissertações de mestrado e teses de doutorado. Pode-se notar que o input exógeno é o recurso utilizado por cada programa na operalização do seu processo. Os produtos intermediários deste processo são as dissertações de mestrado e teses de doutorado, que são as variáveis de entrada (inputs) do segundo estágio. Os outputs finais do processo são as publicações do programa em revistas científicas avaliadas e bem classificadas no QUALIS e consideradas nos critérios de avaliação dos programas de pósgraduação pela CAPES. Foi considerado ainda o mesmo critério de saturação considerado pela CAPES onde o número de máximo de publicações em cada categoria de periódico é o triplo do número de docentes.

Considerando-se que o programa tenha critérios definidos para credenciar professores é razoável supor que, aumentando o número de professores aumente proporcionalmente o número de publicações e é possível também admitir mais alunos, de forma proporcional, pois há mais orientadores. Desta forma, é respeitado o axioma do raio ilimitado que é condição para usar o modelo CCR, com retorno constante de escala, não importando se as instituições são ou não de tamanho semelhante. Notea ainda que o usos do modelo CCR está também coerente com a avaliação da CAPES, que faz uso de simples ratios, o que equivale a um modelo CCR simplificado

Como o objetivo dos programas deve ser o aumento de suas publicações, o modelo deveria ser orientado à output, já que não faz sentido também reduzir o input número de docentes. No entanto, como o modelo é de retorno constante de escala, não é necessário definir a orientação pois, neste caso, as duas fornecem os mesmos índices de eficiência.

Em (11) é apresentado o modelo utilizado neste trabalho. Este modelo é uma evolução do modelo de Kao and Hwang (2008) apresentado em (9), onde a restrição $\bigsqcup_{r=1}^{s} u_{r} \cdot y_{r j} \square \square_{i=1}^{m} v_{i} \cdot x_{i j} \square 0, j=1,2, \ldots, n$. foi retirada, pois a mesma é redundante como demonstrado por Kao (2009) e Chen et al. (2009).

$$
\begin{aligned}
& E_{0}=\max \prod_{r=1}^{7} u_{r} \cdot y_{r 0} \\
& \text { s.t. } v_{1} \cdot x_{10}=1 \\
& \prod_{d=1}^{2} w_{d} \cdot z_{d j} \square v_{1} \cdot x_{1 j} \square 0, \square j \\
& \prod_{r=1}^{7} u_{r} \cdot y_{r j} \square \prod_{d=1}^{2} w_{d} \cdot z_{d j} \square 0, \square j \\
& u_{r}, v_{1}, w_{d} \square \square, \square r ; \square d .
\end{aligned}
$$

As eficiências individuais das DMUs nos estágios 1 e 2 são dadas, respectivamente, pelas equações (12) e (13) enquanto que a eficiência global é calculada pela equação (14). 


$$
\begin{aligned}
& E_{0}^{1}=\frac{\prod_{d=1}^{2} w_{d}^{*} \cdot z_{d 0}}{v_{1}^{*} \cdot x_{10}} \\
& E_{0}^{2}=\frac{\prod_{r=1}^{7} u_{r}^{*} \cdot y_{r 0}}{\prod_{d=1}^{2} w_{d}^{*} \cdot z_{d 0}} \\
& E_{0}=E_{0}^{1} x E_{0}^{2}
\end{aligned}
$$

Foram utilizados os dados do último triênio de avaliação (2010-2012), divulgados pela Capes e, assim, avaliados 42 programas de pós-graduação relacionados na Tabela 1. É importante destacar que foi desconsiderado apenas o programa de doutorado de Engenharia de Produção e Sistemas

\begin{tabular}{|c|c|c|c|c|c|c|c|c|c|c|c|}
\hline \multirow{3}{*}{$\begin{array}{c}\text { Sigla } \\
\text { Instituição }\end{array}$} & \multirow{3}{*}{ Nome } & \multirow{2}{*}{ 遶 } & \multicolumn{2}{|c|}{$\begin{array}{c}\text { Teses e } \\
\text { Dissertações }\end{array}$} & \multicolumn{7}{|c|}{$\begin{array}{l}\text { Artigos completos publicados em } \\
\text { periódicos técnico-científicos }\end{array}$} \\
\hline & & & Te & Di & A1 & A2 & B1 & B2 & B3 & B4 & B5 \\
\hline & & DOCENTES & $\begin{array}{l}\text { 踢 } \\
\text { 至 }\end{array}$ & 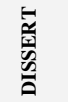 & $\underset{\frac{\pi}{4}}{\bar{z}}$ & 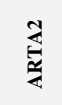 & $\overrightarrow{\underline{\theta}}$ & $\underset{\mathscr{C}}{\tilde{z}}$ & $\underset{\tilde{z}}{\tilde{z}}$ & $\begin{array}{l}\text { 萑 } \\
\text { 总 }\end{array}$ & 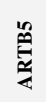 \\
\hline $\begin{array}{l}\text { UNESP } \\
\text { GUAR }\end{array}$ & ENGENHARIA MECÂNICA & 34 & 80 & 119 & 43 & 46 & 70 & 57 & 12 & 43 & 58 \\
\hline UNICAMP & ENGENHARIA MECÂNICA & 59 & 80 & 193 & 77 & 64 & 59 & 43 & 18 & 16 & 15 \\
\hline UFSC & ENGENHARIA DE PRODUÇÃO & 27 & 77 & 48 & 6 & 21 & 43 & 67 & 81 & 81 & 81 \\
\hline ITA & $\begin{array}{l}\text { ENGENHARIA AERONÂUTICA E } \\
\text { MECÂNICA }\end{array}$ & 70 & 65 & 198 & 57 & 69 & 96 & 34 & 6 & 71 & 64 \\
\hline UFSCAR & ENGENHARIA DE PRODUÇÃO & 21 & 64 & 100 & 11 & 16 & 12 & 63 & 30 & 25 & 45 \\
\hline UFSC & ENGENHARIA MECÂNICA & 46 & 59 & 143 & 75 & 29 & 51 & 20 & 12 & 15 & 19 \\
\hline UFRJ & ENGENHARIA DE PRODUÇÃO & 21 & 57 & 127 & 17 & 27 & 44 & 19 & 36 & 29 & 16 \\
\hline USP & ENGENHARIA MECÂNICA & 46 & 57 & 80 & 69 & 52 & 44 & 30 & 8 & 24 & 7 \\
\hline USP/SC & ENGENHARIA MECÂNICA & 41 & 51 & 93 & 36 & 35 & 62 & 11 & 10 & 32 & 13 \\
\hline UFPB/J.P. & ENGENHARIA MECÂNICA & 25 & 43 & 43 & 12 & 8 & 15 & 11 & 4 & 0 & 11 \\
\hline USP/SC & ENGENHARIA DE PRODUÇÃO & 17 & 40 & 63 & 9 & 11 & 32 & 23 & 20 & 29 & 43 \\
\hline UFRJ & ENGENHARIA MECÂNICA & 24 & 38 & 66 & 50 & 19 & 46 & 3 & 4 & 13 & 6 \\
\hline UFRGS & ENGENHARIA DE PRODUÇÃO & 12 & 36 & 42 & 5 & 17 & 14 & 27 & 36 & 36 & 19 \\
\hline UFU & ENGENHARIA MECÂNICA & 22 & 34 & 69 & 23 & 13 & 33 & 30 & 10 & 7 & 27 \\
\hline UFRJ & PLANEJAMENTO ENERGÉTICO & 12 & 33 & 59 & 39 & 16 & 4 & 4 & 11 & 2 & 3 \\
\hline UFRGS & ENGENHARIA MECÂNICA & 29 & 32 & 75 & 37 & 28 & 28 & 12 & 7 & 29 & 30 \\
\hline UNIP & ENGENHARIA DE PRODUÇÃO & 11 & 29 & 43 & 4 & 12 & 22 & 3 & 10 & 12 & 31 \\
\hline USP & ENGENHARIA DE PRODUÇÃO & 28 & 29 & 26 & 6 & 7 & 8 & 37 & 13 & 27 & 16 \\
\hline UFMG & ENGENHARIA MECÂNICA & 27 & 28 & 51 & 38 & 13 & 39 & 12 & 12 & 11 & 18 \\
\hline UFRJ & ENGENHARIA OCEÂNICA & 17 & 27 & 86 & 9 & 13 & 3 & 6 & 6 & 6 & 2 \\
\hline UNIMEP & ENGENHARIA DE PRODUÇÃO & 11 & 25 & 25 & 5 & 1 & 1 & 8 & 3 & 6 & 27 \\
\hline PUC-RIO & ENGENHARIA MECÂNICA & 18 & 25 & 86 & 49 & 30 & 31 & 8 & 1 & 5 & 2 \\
\hline INPE & ENGENHARIA E TECNOLOGIA ESPACIAIS & 45 & 23 & 53 & 46 & 36 & 56 & 26 & 1 & 23 & 13 \\
\hline UFPR & ENGENHARIA MECÂNICA & 19 & 22 & 28 & 36 & 22 & 25 & 17 & 12 & 6 & 6 \\
\hline UFRN & CIÊNCIA E ENGENHARIA DE PETRÓLEO & 16 & 22 & 68 & 36 & 28 & 47 & 9 & 14 & 40 & 15 \\
\hline
\end{tabular}
da PUC/PR - Pontifícia Universidade Católica do Paraná, que iniciou o curso em 2011.

Tabela 1 - Dados do modelo 


\begin{tabular}{|l|l|c|c|c|c|c|c|c|c|c|c|}
\hline UFPR & MÉTODOS NUMÉRICOS EM ENGENHARIA & 25 & 22 & 52 & 10 & 14 & 24 & 7 & 17 & 32 & 33 \\
\hline UFPE & ENGENHARIA MECÂNICA & 19 & 20 & 57 & 3 & 8 & 10 & 15 & 1 & 6 & 9 \\
\hline UFRN & ENGENHARIA MECÂNICA & 23 & 20 & 93 & 21 & 14 & 29 & 12 & 10 & 35 & 25 \\
\hline UFPE & ENGENHARIA DE PRODUÇÃO & 13 & 19 & 64 & 12 & 8 & 11 & 19 & 1 & 9 & 12 \\
\hline PUC-RIO & ENGENHARIA DE PRODUÇÃO & 12 & 19 & 61 & 12 & 4 & 15 & 17 & 4 & 17 & 6 \\
\hline UFPA & ENGENHARIA DE RECURSOS NATURAIS & 10 & 17 & 0 & 11 & 6 & 6 & 4 & 2 & 10 & 4 \\
\hline DA AMAZÔNIA & ENGENHARIA MECÂNICA & 17 & 13 & 40 & 51 & 34 & 19 & 14 & 7 & 6 & 2 \\
\hline USP & ENGENHARIA NAVAL E OCEÂNICA & 12 & 13 & 35 & 5 & 15 & 13 & 4 & 1 & 5 & 2 \\
\hline UFF & ENGENHARIA DE PRODUÇÃO & 21 & 12 & 71 & 5 & 4 & 8 & 27 & 11 & 37 & 50 \\
\hline PUC/MG & ENGENHARIA MECÂNICA & 12 & 11 & 45 & 22 & 6 & 6 & 3 & 4 & 4 & 1 \\
\hline UFBA & ENGENHARIA INDUSTRIAL & 20 & 10 & 30 & 24 & 10 & 28 & 14 & 4 & 6 & 11 \\
\hline UNB & CIÊNCIAS MECÂNICAS & 17 & 10 & 34 & 16 & 29 & 10 & 13 & 6 & 4 & 11 \\
\hline UNIFEI & ENGENHARIA MECÂNICA & 17 & 10 & 27 & 16 & 5 & 26 & 7 & 5 & 4 & 7 \\
\hline UENF & ENGENHARIA DE RESERVATÓRIO E DE & 15 & 8 & 28 & 5 & 6 & 13 & 13 & 1 & 2 & 4 \\
\hline PUC/PR & ENGENHACÃO & 12 & 5 & 14 & 17 & 16 & 12 & 2 & 0 & 1 & 10 \\
\hline UNICAMP & CIÊNCIAS E ENGENHARIA DE PETRÓLEO & 15 & 4 & 59 & 4 & 10 & 15 & 5 & 1 & 4 & 5 \\
\hline UNB & SISTEMAS MECATRÔNICOS & 12 & 2 & 26 & 3 & 9 & 5 & 22 & 1 & 6 & 7 \\
\hline
\end{tabular}

\section{RESULTADOS E DISCUSSÕES}

Durante o triênio 2010-2012 estudado, 42 programas de pós-graduação foram analisados, entre mestrados e doutorados. Foi descartado apenas o programa de doutorado de Engenharia de Produção e Sistemas da PUC/PR - Pontifícia Universidade Católica do Paraná, que iniciou o curso em 2011 e, assim, não possui dados neste triênio. A Tabela 2 apresenta as eficiências encontradas com o modelo NDEA multiplicativo relacional de dois estágios utilizado.

Tabela 2 - Resultados

\begin{tabular}{|c|c|c|c|c|}
\hline $\begin{array}{c}\text { Sigla } \\
\text { Instituição }\end{array}$ & Nome & $\begin{array}{c}\text { Eficiência } \\
1^{0} \text { estágio }\end{array}$ & $\begin{array}{l}\text { Eficiência } \\
2^{0} \text { estágio }\end{array}$ & $\begin{array}{c}\text { Eficiência } \\
\text { global }\end{array}$ \\
\hline UNESP/GUAR & ENGENHARIA MECÂNICA & 0,66800 & 0,54159 & 0,36178 \\
\hline UNICAMP & ENGENHARIA MECÂNICA & 0,52810 & 0,40155 & 0,21206 \\
\hline UFSC & ENGENHARIA DE PRODUÇÃO & 0,52838 & 0,99586 & 0,52620 \\
\hline ITA & ENGENHARIA AERONÂUTICA E MECÂNICA & 0,42880 & 0,50118 & 0,21491 \\
\hline UFSCAR & ENGENHARIA DE PRODUÇÃO & 0,89130 & 0,40349 & 0,35963 \\
\hline UFSC & ENGENHARIA MECÂNICA & 0,50118 & 0,40009 & 0,20052 \\
\hline UFRJ & ENGENHARIA DE PRODUÇÃO & 1,00000 & 0,37000 & 0,37000 \\
\hline USP & ENGENHARIA MECÂNICA & 0,33991 & 0,65575 & 0,22290 \\
\hline USP/SC & ENGENHARIA MECÂNICA & 0,40085 & 0,51122 & 0,20492 \\
\hline UFPB/J.P. & ENGENHARIA MECÂNICA & 0,39261 & 0,23503 & 0,09227 \\
\hline USP/SC & ENGENHARIA DE PRODUÇÃO & 0,69150 & 0,53113 & 0,36728 \\
\hline UFRJ & ENGENHARIA MECÂNICA & 0,49457 & 0,51904 & 0,25670 \\
\hline UFRGS & ENGENHARIA DE PRODUÇÃO & 0,74185 & 0,68508 & 0,50823 \\
\hline UFU & ENGENHARIA MECÂNICA & 0,53434 & 0,45480 & 0,24302 \\
\hline UFRJ & PLANEJAMENTO ENERGÉTICO & 0,87500 & 0,44051 & 0,38545 \\
\hline UFRGS & ENGENHARIA MECÂNICA & 0,42110 & 0,52353 & 0,22046 \\
\hline UNIP & ENGENHARIA DE PRODUÇÃO & 0,74704 & 0,42768 & 0,31949 \\
\hline USP & ENGENHARIA DE PRODUÇÃO & 0,22418 & 0,70528 & 0,15811 \\
\hline UFMG & ENGENHARIA MECÂNICA & 0,33394 & 0,62629 & 0,20914 \\
\hline UFRJ & ENGENHARIA OCEÂNICA & 0,75863 & 0,15062 & 0,11426 \\
\hline UNIMEP & ENGENHARIA DE PRODUÇÃO & 0,51877 & 0,33414 & 0,17334 \\
\hline PUC-RIO & ENGENHARIA MECÂNICA & 0,70380 & 0,47045 & 0,33110 \\
\hline INPE & ENGENHARIA E TECNOLOGIA ESPACIAIS & 0,19275 & 0,97903 & 0,18871 \\
\hline UFPR & ENGENHARIA MECÂNICA & 0,30034 & 0,93410 & 0,28055 \\
\hline UFRN & CIÊNCIA E ENGENHARIA DE PETRÓLEO & 0,64198 & 0,76524 & 0,49127 \\
\hline UFPR & MÉTODOS NUMÉRICOS EM ENGENHARIA & 0,33783 & 0,60648 & 0,20488 \\
\hline UFPE & ENGENHARIA MECÂNICA & 0,46253 & 0,22123 & 0,10232 \\
\hline UFRN & ENGENHARIA MECÂNICA & 0,56073 & 0,42386 & 0,23767 \\
\hline UFPE & ENGENHARIA DE PRODUÇÃO & 0,72868 & 0,28614 & 0,20851 \\
\hline PUC-RIO & ENGENHARIA DE PRODUÇÃO & 0,76087 & 0,34140 & 0,25976 \\
\hline
\end{tabular}




\begin{tabular}{|l|l|c|c|c|}
\hline UFPA & ENGENHARIA DE RECURSOS NATURAIS DA AMAZÔNIA & 0,19402 & 0,90807 & 0,17618 \\
\hline UFF & ENGENHARIA MECÂNICA & 0,35582 & 1,00000 & 0,35582 \\
\hline USP & ENGENHARIA NAVAL E OCEÂNICA & 0,45652 & 0,35459 & 0,16188 \\
\hline UFF & ENGENHARIA DE PRODUÇÃO & 0,45109 & 0,56045 & 0,25281 \\
\hline PUC/MG & ENGENHARIA MECÂNICA & 0,53261 & 0,40824 & 0,21743 \\
\hline UFBA & ENGENHARIA INDUSTRIAL & 0,22826 & 0,85101 & 0,19425 \\
\hline UNB & CIÊNCIAS MECÂNICAS & 0,29540 & 0,70169 & 0,20728 \\
\hline UNIFEI & ENGENHARIA MECÂNICA & 0,24840 & 0,67396 & 0,16741 \\
\hline UENF & ENGENHARIA DE RESERVATÓRIO E DE EXPLORAÇÃO & 0,27391 & 0,43980 & 0,12047 \\
\hline PUC/PR & ENGENHARIA MECÂNICA & 0,18071 & 1,00000 & 0,18071 \\
\hline UNICAMP & CIÊNCIAS E ENGENHARIA DE PETRÓLEO & 0,47935 & 0,24503 & 0,11746 \\
\hline UNB & SISTEMAS MECATRÔNICOS & 0,26630 & 0,65029 & 0,17317 \\
\hline
\end{tabular}

De acordo com o resultado encontrado, apenas o programa de Engenharia de Produção da Universidade Federal do Rio de Janeiro (UFRJ) foi eficiente no $1^{\circ}$ estágio. Isto ocorre porque o programa possui um número elevado de dissertações e teses defendidas e, além disso, possui um número baixo de docentes cadastrados ao programa. No entanto, esta eficiência não se mantém alta no $2^{\circ}$ estágio, pois se verifica que a quantidade de publicações não é tão elevada quando de outros programas. Assim, a eficiência global do programa é baixa, apesar do número elevado de defesas, já que o mesmo não consegue publicar estes trabalhos com tanta frequência.

Com relação ao $2^{0}$ estágio, o único programa eficiente foi o de Engenharia Mecânica da Pontifícia Universidade Católica do Paraná (PUC/PR) uma vez que o mesmo apresenta um bom número de publicações em relação ao número de defesas de dissertações e teses. Por outro lado, o mesmo programa possui eficiência baixa no $1^{\circ}$ estágio por possuir um baixo número de defesas, o que implica em uma eficiência global baixa.

O programa com maior eficiência global é o de Engenharia de Produção da Universidade Federal de Santa Catarina (UFSC) por possuir um número elevado de defesas de dissertações e teses e também de publicações. Para exemplificar, foi o único curso que atingiu o valor de saturação de publicações em 03 (três) categorias de revistas (B3, B4 e B5).

Por outro lado, o curso de Engenharia de Produção da Universidade de São Paulo (USP) obteve o menor índice de eficiência global por possuir elevado número de professores no programa e baixo número de publicações.

\section{CONCLUSÕES}

A utilização de um modelo NDEA, em oposição ao clássico DEA, ofereceu a vantagem de decompor o processo de produção dos programas de pós-graduação em 02 (dois) estágios sequenciais, onde a elaboração e defesa de dissertações de mestrado e teses de doutorado foram considerados produtos intermediários na produção dos outputs do processo, ou seja, as publicações em periódicos considerados válidos pela CAPES na avaliação trienal dos programas de pós-graduação, além da avaliação da eficiência associada a cada uma delas. Além disso, em relação aos programas ineficientes, a divisão do processo de produção tornou mais fácil identificar as fontes de ineficiência no processo como um todo.

Note-se que não houve nenhum programa gloalmente eficiente. Este fato pode ser útil em estudos futuros de variáveis explicativas, já que poderá permitir uso de certas regressões que, por motivos técnicos, não podem ser usadas com DEA tradicional.

\section{AGRADECIMENTOS}

Ao CNPq e à Fundación Carolina.

\section{REFERÊNCIAS}

Angulo-Meza, L., Biondi Neto, L., Brandão, L. C., Andrade, F. V. S., Soares de Mello, J. C. C. B. e Coelho, P. H. G., Modelling with self-organising maps and data envelopment 
analysis: A case study in educational evaluation (Eds),_Self organizing maps, new achievements, Intech, Vienna, 71-88, 2011.

Angulo Meza, L., Soares de Mello, J. C. C. B. e Gomes Junior, S. F., Benchmarking Distance Learning Centers with a Multiobjective Data Envelopment Analysis Model em Holtzman, Y. (Eds),_Advanced Topics in Applied Operations Management, Intech, 183200, 2012.

Banker, R. D., Charnes, A. e Cooper, W. W. (1984), Some models for estimating technical scale inefficiencies in data envelopment analysis, Management Science, 30(9), 10781092.

Boclin, R. (1999), Indicadores de desempenho: Novas estratégias da educação superior, Ensaio: Avaliação e Políticas Públicas em Educação, 7, 299-308.

Bougnol, M.-L. e Dulá, J. H. (2006), Validating DEA as a ranking tool: An application of DEA to assess performance in higher education, Annals of Operations Research, 145(1), 339-365.

Charnes, A., Cooper, W. W. e Rhodes, E. (1978), Measuring the efficiency of decisionmaking units, European Journal of Operational Research, 2, 429-444.

Chen, Y., Cook, W. D., Li, N. e Zhu, J. (2009), Additive efficiency decomposition in two-stage DEA, European Journal of Operational Research, 196(3), 1170-1176.

Chen, Y. e Zhu, J. (2004), Measuring Information Technology's Indirect Impact on Firm Performance, Information Technology and Management 5(1-2), 9-22.

Chilingerian, J. A. e Sherman, H. D., Health care applications: From hospitals to physicians, from productive efficiency to quality frontiers em Cooper, W. W., Seiford, L. M. e Zhu, J. (Eds),_Handbook on data envelopment analysis, Springer, Boston, 2004.

Cooper, W. W., Seiford, L. e Tone, K., Data envelopment analysis: A comprehensive text with models, applications, references and DEA-solver software, Kluwer, Boston, 2000.

de França, J. M. F., de Figueiredo, J. N. e dos Santos Lapa, J. (2010), A DEA methodology to evaluate the impact of information asymmetry on the efficiency of not-for-profit organizations with an application to higher education in Brazil, Annals of Operations Research, 173(1), 39-56.

Färe, R. e Grosskopf, S. (2000), Network DEA, Socio-Economic Planning Sciences, 34(1), 35 49.

Gomes Junior, S. F., Soares de Mello, J. C. C. B. e Angulo-Meza, L. (2013), DEA nonradial efficiency based on vector properties, International Transactions in Operational Research, 20(3), 341-364.

Kao, C. (2009), Efficiency decomposition in network data envelopment analysis: A relational model, European Journal of Operational Research, 192(3), 949-962.

Kao, C. e Hwang, S. N. (2008), Efficiency decomposition in two-stage data envelopment analysis: An application to non-life insurance companies in Taiwan, European Journal of Operational Research, 185(1), 418-429.

Moreno, P. e Lozano, S. (2014), A network DEA assessment of team efficiency in the NBA, Annals of Operations Research, 214(1), 99-124.

Narasimhan, R., Talluri, S. e Das, A. (2004), Exploring flexibility and execution competencies of manufacturing firms, Journal of Operations Management, 22, 91106.

Seiford, L. M. e Zhu, J. (1999), Profitability and marketability of the top 55 U.S. commercial banks, Management Science, 45(9), 1270-1288.

Sexton, T. R. e Lewis, H. F. (2003), Two-stage DEA: An application to major league baseball, Journal of Productivity Analysis, 19, 227-249. 
Soares de Mello, J. C. C. B., Gomes, E. G., Angulo-Meza, L., Soares de Mello, M. H. C. e Soares de Mello, A. J. R. (2006), Engineering Post-Graduate Programmes: A Quality and Productivity Analysis, Studies in Educational Evaluation, 32, 136-152.

Thanassoulis, E., Portela, M. C. e Allen, R., Incorporating Value Judgments in DEA em Cooper, W. W., Seiford, L. M. e Zhu, J. (Eds),_Handbook on Data Envelopment Analysis, Kluwer Academic Publishers, New York, Boston, Dordrecht, London, Moscow, 99138, 2004.

Thieme, C., Giménez, V. e Prior, D. (2012), A comparative analysis of the efficiency of national education systems, Asia Pacific Education Review, 13(1), 1-5.

Tone, K. e Tsutsui, M. (2009), Network DEA: A slacks-based measure approach, European Journal of Operational Research, 197(1), 243-252.

Tyagi, P., Yadav, S. P. e Singh, S. P. (2009), Relative performance of academic departments using DEA with sensitivity analysis, Evaluation and Program Planning, 32(2), 168177.

Zhu, J. (2000), Multi-factor performance measure model with an application to Fortune 500 companies, European Journal of Operational Research, 123(1), 105-124. 